\title{
Frequency Comb Generation by Bloch Gain Induced Giant Kerr Nonlinearity
}

\author{
Nikola Opačak@, ${ }^{*}$ Sandro Dal Cin, Johannes Hillbrand®, and Benedikt Schwarz $\circledast^{\dagger}$ \\ Institute of Solid State Electronics, TU Wien, Gusshausstrasse 25-25a, 1040 Vienna, Austria
}

(Received 25 March 2021; accepted 8 July 2021; published 27 August 2021)

\begin{abstract}
Optical nonlinearities are known to coherently couple amplitude and phase of light, which can result in the formation of periodic waveforms. Such waveforms are referred to as optical frequency combs. Here we show that Bloch gain — a nonclassical phenomenon that was first predicted in the 1930s — can play an essential role in comb formation. We develop a self-consistent theoretical model that considers all aspects of comb dynamics: band structure, electron transport, and cavity dynamics. In quantum cascade lasers, Bloch gain gives rise to a giant Kerr nonlinearity, which enables frequency modulated combs and serves as the physical origin of the linewidth enhancement factor. Bloch gain also triggers the formation of solitonlike structures in ring resonators, paving the way toward electrically driven Kerr combs.
\end{abstract}

DOI: 10.1103/PhysRevLett.127.093902

Bloch and Zener predicted charge oscillations in a periodic potential under a constant electric field in the 1930s [1,2], a phenomenon which is referred to as Bloch oscillations. It attracted researchers ever since due to the coupling with electromagnetic waves, potentially offering new sources of radiation [3]. The motion of electrons in a periodic crystal lattice is governed by the energy-momentum relation within a Brillouin zone (BZ) [4]. A constant electric field accelerates the electrons toward the edge of the BZ, where they experience Bragg reflection, resulting in oscillations. In semiconductor superlattices [Fig. 1(a)], the width of the BZ is narrow and electrons can complete multiple oscillation cycles within their lifetime [5]. Ktitorov et al.[6] predicted tunable optical Bloch gain arising from these oscillations, which was subsequently verified in a GaAs-AlGaAs superlattice [7]. The gain exists even without population inversion-a necessary ingredient in the case of a classical harmonic oscillator [Fig. (1b)]. Moreover, Bloch gain possesses an S-shaped profile [Fig. 1 (a)], known as the dispersive gain [7]. This unique spectral response, contrasted by the symmetric Lorentzian gain of a harmonic oscillator, is a fingerprint feature of the Bloch gain. Analogous observations appear also in other physical systems: Josephson junctions [8], Bose-Einstein condensates [9], parity-time-symmetric potentials [10], optical [11] and acoustic [12] waves, and in synthetic dimensions in photonics [13].

More recently, quantum-mechanical treatments of the Bloch gain were developed in the density matrix [14] and

Published by the American Physical Society under the terms of the Creative Commons Attribution 4.0 International license. Further distribution of this work must maintain attribution to the author(s) and the published article's title, journal citation, and DOI. the Green's function [15] formalisms. They generalized the concept of the Bloch gain and showed that it can appear between any two states (subbands) in semiconductor heterostructures, such as quantum cascade lasers (QCLs). QCLs are unipolar lasers [16], which emit in the midinfrared [17] and terahertz [18] spectral regions [Fig. 1(c)]. The gain bandwidth of QCLs is broadened by elastic scattering processes beyond its natural limit defined with the carrier lifetimes $[19,20]$. An accompanying effect of these processes, neglected by most researchers so far, is scattering-assisted optical transitions that yield the Bloch gain [14]. The latter was also experimentally confirmed in QCLs [21].

In this Letter, we conduct a theoretical study of the Bloch gain and its influence on the laser dynamics. A meticulous simulation tool is developed which self-consistently couples every aspect of QCL operation-from electronic band structure and charge transport to the light spatiotemporal evolution within the laser cavity. We show that a dominant Bloch gain contribution is present in any operating QCL and causes a giant Kerr nonlinearity at the laser wavelength. The induced nonlinearity plays an essential role in the laser cavity dynamics as it is a requirement for self-starting optical frequency combs [22]. Bloch gain is not only the reason why frequency modulated (FM) comb formation is predominantly found in dispersion compensated cavities $[23,24]$, but it also allows one to tune the laser into the phase turbulence regime. This can trigger the generation of solitonlike structures [25,26], establishing a bridge between semiconductor QCL lasers and Kerr microresonators [27].

The spectral response of the laser active region is described by its complex susceptibility: $\chi=\chi_{R}+i \chi_{I}$. The gain is defined as $g=\omega \chi_{I} / n_{r} c$ with $\omega$ being the frequency, $n_{r}$ the refractive index, and $c$ the speed of light. The susceptibility that arises from any two subbands $u$ and $l$ is calculated as [14] 

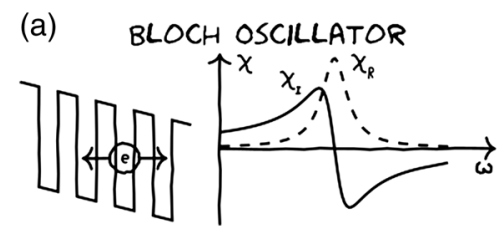

(b)

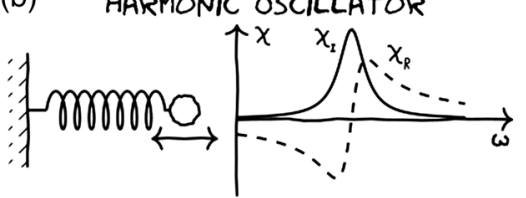

(c) QUANTUM CASCADE LASER

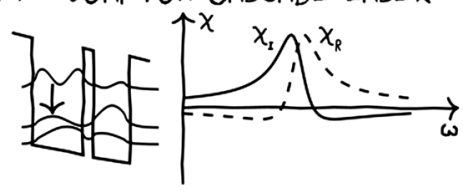

FIG. 1. Illustration of different systems and their optical susceptibilities. The complex susceptibility of a QCL can be represented as a sum of both Bloch and harmonic contributions.

$$
\begin{aligned}
& \chi(\omega)=\frac{\mu_{u l}^{2} \omega_{0}^{2}}{\varepsilon_{0} \omega^{2}} \sum_{k}\left[\frac{f_{u}(k)-f_{l}(k)}{(\hbar \omega-\Delta W(k))-i \gamma(k)}+\right. \\
& \left.i \frac{\gamma_{u}(k)\left(f_{u}\left(k_{-}\right)-f_{u}(k)\right)-\gamma_{l}(k)\left(f_{l}\left(k_{+}\right)-f_{l}(k)\right)}{(\hbar \omega-\Delta W(k))((\hbar \omega-\Delta W(k))-i \gamma(k))}\right] .
\end{aligned}
$$

The total susceptibility in Eq. (1) comprises two components. The usual harmonic contribution is given by the first term within the large parentheses. It depends on the population inversion $f_{u}(k)-f_{l}(k)$ and yields a Lorentzian gainshape. The dipole matrix element is $\mu_{u l}, \varepsilon_{0}$ is the vacuum permittivity, $f(k)$ and $\gamma(k)$ are the electron distribution and broadening at wave vector $k$, and $\Delta W(k)=$ $W_{u}(k)-W_{l}(k)$ is the resonant transition energy, where $\Delta W_{0}=\Delta W(k=0)=\hbar \omega_{0}$. The highlighted second term in Eq. (1) is more intriguing. It generates the Bloch gain by allowing optical transitions between states with different wave vectors. The level broadenings are $\gamma_{u, l}$, where $\gamma=$ $\gamma_{u}+\gamma_{l}$ [20], and the in-plane momenta of the final states are $k_{ \pm}^{2}=\left(m_{l, u} / m_{u, l}\right) k^{2} \pm\left(2 m_{l, u} / \hbar^{2}\right)\left(\Delta W_{0}-\hbar \omega\right)$. A thorough analysis is given in the Supplemental Material, Sec. 1 [28].

While Eq. (1) provides a perturbative analytic treatment of the Bloch gain, the origin of the dispersive spectral shape is not intuitive. Equation (1) significantly reduces its complexity by assuming electron distributions in the frame of Boltzmann statistics. Following the derivation presented in the Supplemental Material, Sec. 1.1 [28], we obtain

$$
\begin{aligned}
\chi(\omega) & =\frac{\mu_{u l}^{2} \omega_{0}^{2}}{\varepsilon_{0} L_{p} \omega^{2}} \frac{\left(n_{u}-n_{l}\right)+i \frac{\gamma}{2 k_{B} T}\left(n_{u}+n_{l}\right)}{\hbar \omega-\Delta W_{0}-i \gamma} \\
& =\frac{\mu_{u l}^{2} \omega_{0}^{2}}{\varepsilon_{0} L_{p} \omega^{2}} \frac{n_{u}-n_{l}}{\hbar \omega-\Delta W_{0}-i \gamma}(1+i b),
\end{aligned}
$$

where $L_{p}$ is the period length, $k_{B}$ is the Boltzmann constant, $T$ is the temperature, and $n_{u, l}$ are the electron sheet densities. Equation (2) explains the origin of the Bloch gain, which is proportional to the highlighted term. Contrary to the harmonic susceptibility, it is not dependent on the population inversion $\left(n_{u}-n_{l}\right)$ but rather on the population sum $\left(n_{u}+n_{l}\right)$. The dispersive gainshape appears due to the imaginary value of the highlighted terms. They induce a $\pi / 2$ phase shift and exchange the shapes of $\chi_{R}$ and $\chi_{I}$ [Figs. 1(a) and 1(b)]. The factor $b$ in
Eq. (2) captures the impact of the Bloch gain. It deviates the total gainshape from a Lorentzian curve and causes spectral asymmetry. Subband nonparabolicity, which is known to induce a similar behavior, has a weaker effect. Most importantly, Eq. (2) allows for the straightforward implementation of Bloch gain in any carrier transport model, unlike Eq. (1), which requires $k$-space resolved approaches.

With the aim of quantitatively assessing the influence of the Bloch gain on the laser dynamics, our model is employed with a reference midinfrared QCL two-phonon design [30]. For details about our band structure and charge transport model, see the Supplemental Material [28]. The calculated conduction-band profile with probability densities of the states and the electron density are shown in the Supplemental Material, Sec. 4.1 [28]. The calculation of the optical gain for the lasing transition follows from Eq. (1). Its unsaturated value is shown in Fig. 2(a). The Bloch gain induces an asymmetric total gainshape and a redshift of the peak. However, the unsaturated gain asymmetry conveys only a fraction of what happens above the laser threshold. In the usual harmonic description, the emission of light depletes the population inversion until the gain saturates to the threshold value, while $\chi_{R}$ remains zero at the gain peak [Fig. 2(b)]. On the other hand, the Bloch gain is independent of the population inversion and thus remains mostly unaffected. As the harmonic gain fades away with stronger light intensity, the Bloch contribution prevails and results in an increasingly asymmetrical gain accompanied by a redshift and nonzero $\chi_{R}$ at the gain peak [Fig. 2(c)]. Intriguingly, a negative global population inversion is required to completely diminish the total gain (Supplemental Material, Sec. 4.2 [28]). Figure 2(d) shows the saturated gain for three different current densities $J$. The gain peak is blueshifted due to the quantum-confined Stark effect and, more importantly, the asymmetry increases toward the dispersive shape.

Gain asymmetry has historically been analyzed in the context of the laser linewidth broadening. It was treated with the linewidth enhancement factor (LEF) [31], defined as $\mathrm{LEF}=-\left(\partial \chi_{R} / \partial N\right) /\left(\partial \chi_{I} / \partial N\right)$, where $N$ is the carrier population. In interband lasers, the gain asymmetry and LEF dominantly originate from the opposite curvature of the valence and conduction band. Since subbands in a QCL have similar curvatures, the LEF was expected to vanish. Interestingly, nonzero experimental values were obtained mostly between -0.5 and $1.5[32,33]$. We explain this with the gain asymmetry in QCLs that is dominantly caused by 


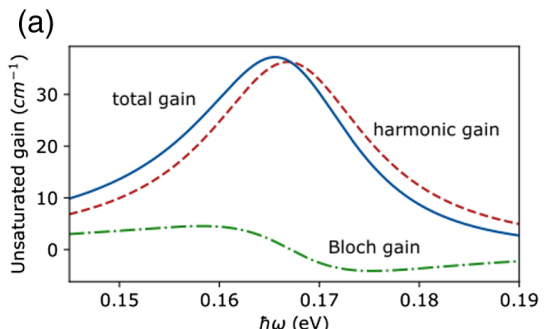

(d)

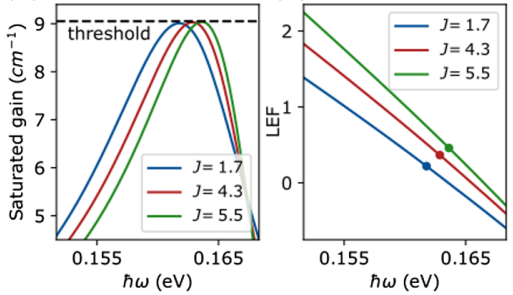

(b)
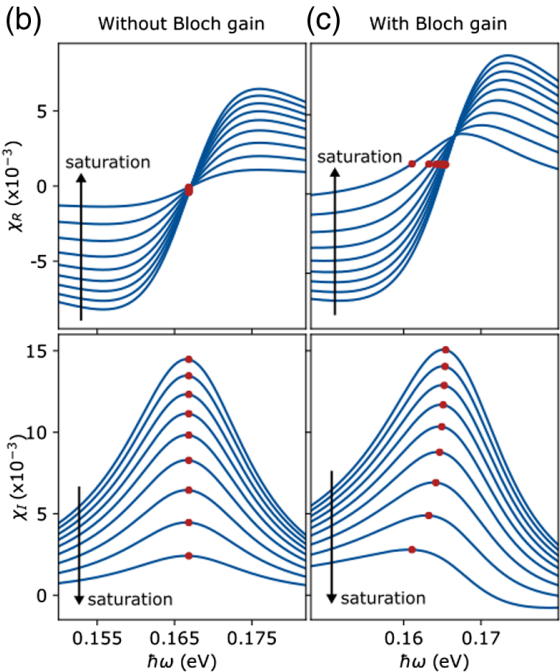
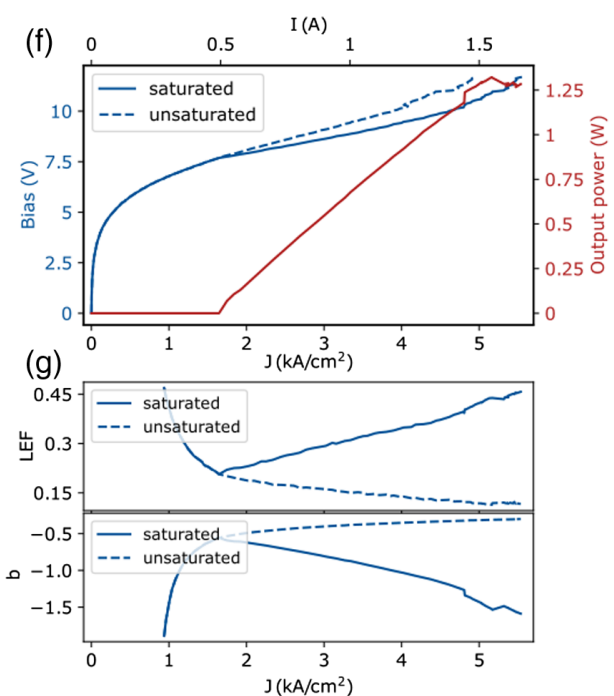

FIG. 2. Bloch gain in QCLs. (a) Unsaturated optical gain from Eq. (1) represented as the sum of the harmonic Lorentzian contribution and dispersive Bloch gain. (b)-(c) Effect of saturation on real $\chi_{R}$ and imaginary part $\chi_{I}$ of the optical susceptibility, whether the Bloch gain is included or not. Red dots indicate values at gain peak, and arrows show the direction of the increasing intensity. Bloch gain induces asymmetric gain, redshift, and nonzero values of $\chi_{R}$ at the gain peak. (d) Saturated gain clamped to the threshold for three values of the current density $J\left(\mathrm{kA} / \mathrm{cm}^{2}\right)$. (e) Spectrally calculated LEF for the same current densities as in (d). Values at the gain peak are indicated with dots. (f) LIV characteristic of the QCL design [30] operating in continuous wave at room temperature. (g) Dependence of LEF and factor $b$ from Eq. (2) on the current density. A linearlike dependence of both values is observed.

the Bloch gain. Furthermore, the LEF is frequency dependent, which yields a large range of values [Fig. 2(e)]. This is in excellent agreement with a recent measurement of the spectrally resolved LEF above lasing threshold, obtained by a novel technique [34]. A measurement of the LEF depending on the light intensity (Supplemental Material, Fig. 7 [28]) would further prove the key role of the Bloch gain. Elimination of the Bloch gain in QCLs yields a symmetric gain profile and vanishing LEF (Supplemental Material, Sec. 4.2 [28]). Figure 2(f) shows the simulated light-current-voltage (LIV) characteristic with the lasing threshold at around $J=1.6 \mathrm{kA} / \mathrm{cm}^{2}$ and rollover at $J=$ $5.5 \mathrm{kA} / \mathrm{cm}^{2}$ [30]. The calculated values of the LEF and factor $b$ at the gain peak are shown in Fig. 2(g). While the population inversion is clamped, the population sum increases with the current in Eq. (2). This leads to a linearlike dependence of the LEF and factor $b$ on $J$, which matches observations found in the literature $[32,33,35]$. The impact of the gain saturation is underlined yet again, as the saturated values notably break off from the unsaturated ones.

The asymmetric gainshape causes population-dependent changes of the susceptibility in the active region [36], leading to a dependence of the gain and refractive index on the intensity. Because of the ultrafast dynamics of the QCL, Bloch gain therefore gives rise to a giant Kerr nonlinearity. Based on the saturation analysis of $\chi$ in Supplemental Material, Sec. 3 [28], we calculate the resonant Kerr contribution due to Bloch gain to be in the range of $10^{-15} \mathrm{~m}^{2} / \mathrm{W}$, which is 2 orders of magnitude larger than the highest bulk values [37]. The value of the Kerr nonlinearity can be tailored by changing the laser heterostructure design.

Optical nonlinearities couple the amplitude and phase of the laser field and give rise to coherent processes such as frequency comb formation. Frequency combs are waveforms whose spectra consist of equidistant modes with a fixed phase relation $[38,39]$. Although historically their formation relied on the emission of short pulses [40], recently a new type of FM combs is blossoming. They are self-starting and appear in numerous Fabry-Pérot laser types such as QCLs [41], interband cascade lasers [42], quantum dot lasers [43], and laser diodes [44]. The fascinating property of FM combs, which distinguishes them from other frequency combs, is an almost constant intensity accompanied by a linear frequency chirp [45]. This unique behavior was explained in [22] as a result of the group velocity dispersion (GVD) or a Kerr nonlinearity, thus underlining the key role of the Bloch gain in FM comb formation.

In order to quantitatively study the frequency comb dynamics, we conduct spatiotemporal simulations of the intracavity field based on a master equation approach [22]. We describe the gain shape asymmetry through the parameter $b$ from Eq. (2). Its population dependence can be accurately modeled as a function of the current density $J$ and the laser intensity $I$ (Supplemental Material, Eq. (42) [28]). This allows a self-consistent implementation of the Bloch gain into the master equation: 


$$
\begin{gathered}
\left(\frac{n}{c} \partial_{t} \pm \partial_{z}\right) E_{ \pm}=\frac{g}{2} \frac{1+i b}{1+i \xi}\left[E_{ \pm}-\tilde{T}_{2} \partial_{t} E_{ \pm}+\tilde{T}_{2}^{2} \partial_{t}^{2} E_{ \pm}\right] \\
-\frac{g T_{g}}{2 T_{1} I_{\mathrm{sat}}} \frac{1+i b}{1+i \xi}\left[\left|E_{\mp}\right|^{2} E_{ \pm}-\left(\tilde{T}_{2}+T_{\mathrm{g}}\right)\left|E_{\mp}\right|^{2} \partial_{t} E_{ \pm}\right. \\
\left.-\left(\tilde{T}_{2}+T_{\mathrm{g}}\right) E_{ \pm} E_{\mp} \partial_{t} E_{\mp}^{*}-\tilde{T}_{2} E_{ \pm} E_{\mp}^{*} \partial_{t} E_{\mp}\right]-\frac{\alpha_{w}}{2} E_{ \pm},
\end{gathered}
$$

where $E \pm$ are the right and left propagating field envelopes, $T_{1}, T_{2}$, and $T_{g}$ are the recovery times of the gain, polarization and the population grating, $\alpha_{w}$ is the waveguide loss, $g$ is the saturated gain, $I_{\text {sat }}$ is the saturation intensity, and $I=\left|E_{+}\right|^{2}+\left|E_{-}\right|^{2}$ is the normalized intensity. The Bloch gain enters the equation through terms $b$, $\xi(b)$, and $\tilde{T}_{2}(b)$. A detailed derivation is presented in the Supplemental Material, Sec. 2.3 [28], along with the analysis for interband lasers with slower dynamics. Boundary conditions for a Fabry-Pérot cavity are $E_{-}(L)=$ $\sqrt{R_{r}} E_{+}(L)$ and $E_{+}(0)=\sqrt{R_{l}} E_{-}(0)$, and for a ring cavity $E_{ \pm}(0)=E_{ \pm}(L)$, where $L$ is the cavity length and $R_{l, r}$ are the reflectivities of the left and right facet.

Temporal evolution of the light intensity in a Fabry-Pérot QCL for one bias point is shown in Fig. 3(a). The inclusion of the Bloch gain leads to a periodic waveform after 6000 round trips and the formation of an FM comb, which is fully characterized in the Supplemental Material, Sec. 4.2 [28]. Conversely, the intensity evolves chaotically in the absence of a locking mechanism provided by the Bloch gain induced Kerr nonlinearity. By extracting the scattering rates from the transport model, we are able to accurately simulate the intracavity dynamics from the laser threshold to rollover [Fig. 3(b)]. The laser is in the single-mode regime near the threshold and significantly broadens its spectrum with the current increase. The key role of the Bloch gain is clearly visible, as it leads to an FM comb operation over the entire bias range. This is indicated by the autocorrelation value equal to 1 in Fig. 3(b). In sharp contrast, the pure harmonic gain results in unlocked states with the autocorrelation smaller than unity and chaotic spectra. This validates the Bloch gain induced giant Kerr nonlinearity as an efficient locking mechanism and explains why FM combs in QCLs have mostly been found in GVD compensated cavities $[23,24,45]$. A nonzero GVD yields an unlocked state for most of the bias range (Supplemental Material, Sec. 4.4 [28]), in accordance with the literature [24].

Linking the physics of FM combs to Bloch gain induced giant Kerr nonlinearity suggests a connection to the Kerr combs in microresonators [27]. They represent passive media, where pumping is achieved through an external injection of a monochromatic laser and the gain stems from the Kerr nonlinearity of the bulk crystal. Through a cascaded parametric process, the injected wave induces the appearance of side modes, giving rise to phase-locked frequency combs in the form of temporal solitons [46]. QCL combs in ring cavities [Fig. 4(a)] have recently been
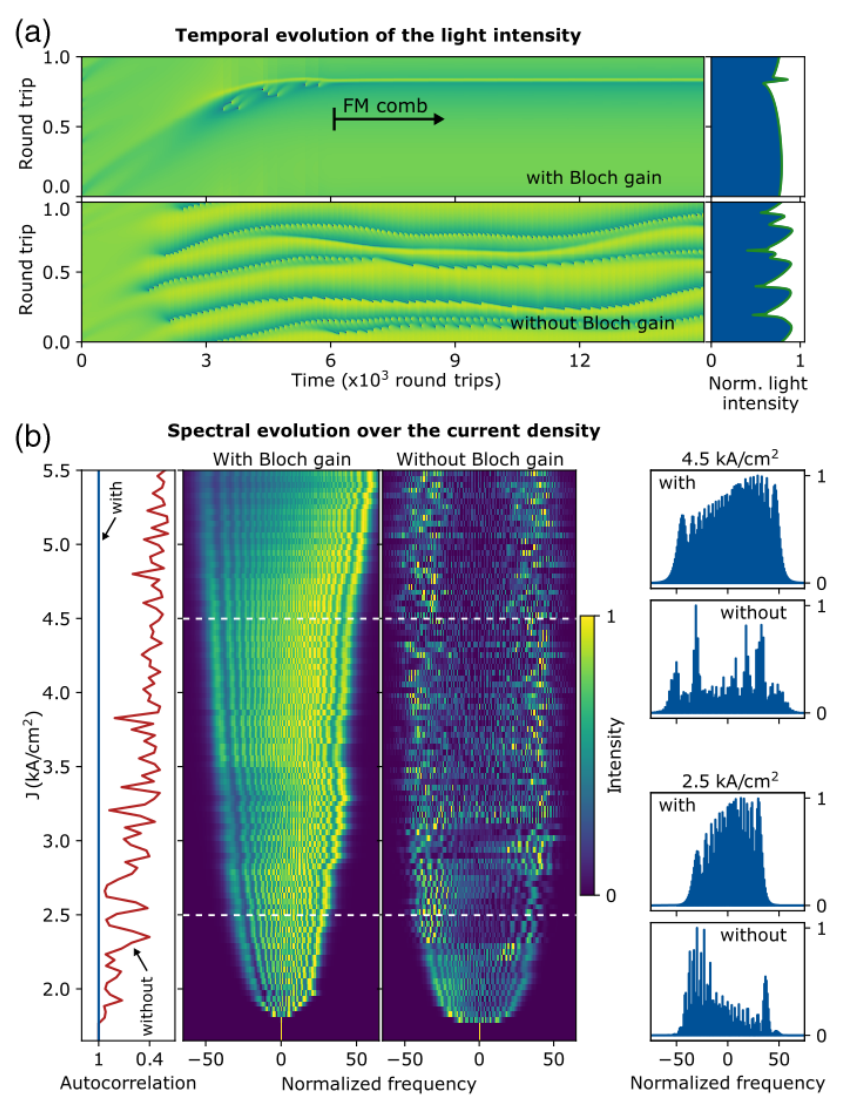

FIG. 3. FM combs in a Fabry-Pérot cavity. (a) Temporal evolution of the intensity. Excluding Bloch gain results in an unlocked state (bottom). Including Bloch gain leads to a stable FM comb (top). The waveforms in the last round trip are shown on the right. (b) Evolution of the intensity spectrum with the increasing current density $J$. Near threshold, the spectrum consists of a single mode and broadens with the increase of $J$. The Bloch gain induced Kerr nonlinearity forms a locked FM comb over the entire range of $J$, as is seen from the autocorrelation value equal to unity (blue line on the left). Neglecting the Bloch contribution leads to unlocked states with the autocorrelation value smaller than unity (red line on the left). The four spectra on the right are taken at $2.5 \mathrm{kA} / \mathrm{cm}^{2}$ and $4.5 \mathrm{kA} / \mathrm{cm}^{2}$, indicated by the white dashed lines.

shown to possess several similarities with Kerr microresonators $[25,26]$. Within the framework of the GinzburgLandau formalism [47], it was demonstrated that a singlemode operation is destabilized by the phase turbulence. The latter is controlled with the laser nonlinearity to induce multimode emission with a hyperbolic secant (sech) spectrum type [Fig. 4(b)]. The nonlinearity can trigger the formation of localized structures in the waveform [Fig. 4(c)], which are related to dissipative Kerr solitons. In the absence of the Bloch gain, the laser operates in a single-mode regime with constant intensity. The study in [48] demonstrated that the Kerr microresonators and ring QCLs can both be analyzed within the same theoretical framework and predicted the emission of temporal solitons from a ring QCL with a suitable nonlinearity. 


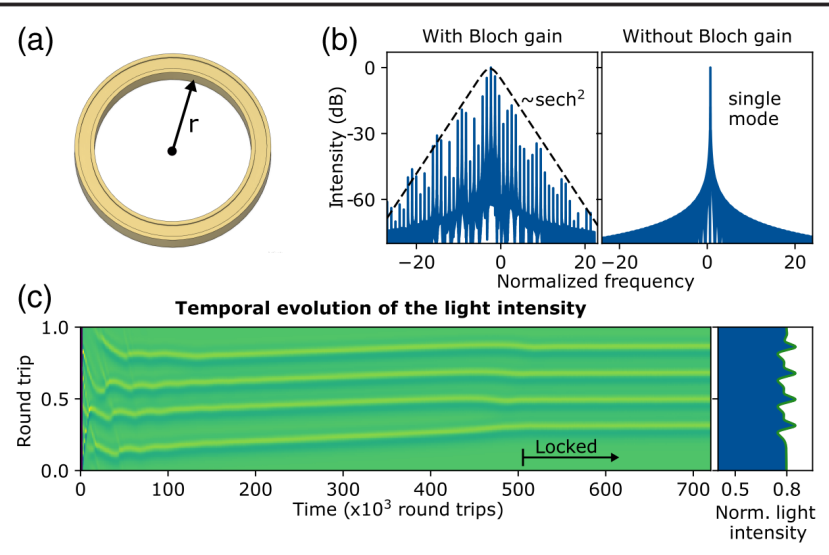

FIG. 4. Spatial patterns in a monolithic ring laser comb. (a) Schematic of a ring cavity laser. (b) Intensity spectra of a ring QCL. Bloch gain leads to a multimode instability through phase turbulence [25]. A sech ${ }^{2}$ envelope is fitted to the spectrum. Elimination of Bloch gain yields single-mode operation. (c) Temporal evolution of the intensity shows an initial turbulent regime that forms a frequency comb after 510000 round trips.

In conclusion, we have shown that the Bloch gain contribution due to intersubband transitions provides a locking mechanism for frequency comb formation. The saturated total gain considerably deviates from a Lorentzian curve toward a dispersive asymmetric shape due to Bloch gain. We capture its nonclassical nature with the master equation to study the spatiotemporal evolution of the laser field and show that FM comb formation in FabryPérot QCLs is triggered by a Bloch gain induced giant Kerr nonlinearity. The Bloch gain acts as a locking mechanism over the entire range of the bias, explaining why FM combs were experimentally observed mostly in GVD compensated cavities. In a ring resonator, the impact of Bloch gain is particularly strong due to low cavity losses and stronger saturation. The induced Kerr nonlinearity can result in comb formation and the emission of solitonic structures, paving the way toward electrically driven Kerr combs in the midinfrared range. As we now know that the Kerr nonlinearity dominantly stems from the Bloch gain, using our model will allow researchers to tailor the nonlinearity in order to optimize frequency comb operation and to discover new states of light.

This project has received funding from the European Research Council (ERC) under the European Union's Horizon 2020 research and innovation program (Grant agreement No. 853014) and European Union's Horizon 2020 research and innovation program (Grant agreement No. 871529). We acknowledge discussions with Alexey Belyanin and Yongrui Wang on the origin of the linewidth enhancement factor, and Gerald Bastard on the secondorder scattering processes.

*nikola.opacak@tuwien.ac.at

†benedikt.schwarz@tuwien.ac.at
[1] F. Bloch, Z. Phys. 52, 555 (1929).

[2] C. Zener, Proc. R. Soc. Ser. A 145, 523 (1934).

[3] C. Waschke, H. G. Roskos, R. Schwedler, K. Leo, H. Kurz, and K. Köhler, Phys. Rev. Lett. 70, 3319 (1993).

[4] N. W. Ashcroft and D. N. Mermin, Solid State Physics (Brooks Cole, Belmont, MA, 1976).

[5] K. Leo, P. H. Bolivar, F. Brüggemann, R. Schwedler, and K. Köhler, Solid State Commun. 84, 943 (1992).

[6] S. A. Ktitorov, G. S. Simin, and V. Y. Sindalovskii, Fiz. Tver. Tela 13, 2230 (1971).

[7] N. Sekine and K. Hirakawa, Phys. Rev. Lett. 94, 057408 (2005).

[8] D. V. Averin, A. B. Zorin, and K. K. Likharev, Sov. Phys. JETP 61, 407 (1985).

[9] M. Ben Dahan, E. Peik, J. Reichel, Y. Castin, and C. Salomon, Phys. Rev. Lett. 76, 4508 (1996).

[10] S. Longhi, Phys. Rev. Lett. 103, 123601 (2009).

[11] T. Pertsch, P. Dannberg, W. Elflein, A. Bräuer, and F. Lederer, Phys. Rev. Lett. 83, 4752 (1999).

[12] H. Sanchis-Alepuz, Y. A. Kosevich, and J. Sánchez-Dehesa, Phys. Rev. Lett. 98, 134301 (2007).

[13] L. Yuan and S. Fan, Optica 3, 1014 (2016).

[14] H. Willenberg, G. H. Döhler, and J. Faist, Phys. Rev. B 67, 085315 (2003).

[15] A. Wacker, Phys. Rev. B 66, 085326 (2002).

[16] J. Faist, F. Capasso, D. L. Sivco, C. Sirtori, A. L. Hutchinson, and A. Y. Cho, Science 264, 553 (1994).

[17] Y. Yao, A. J. Hoffman, and C. F. Gmachl, Nat. Photonics 6, 432 (2012).

[18] B. S. Williams, Nat. Photonics 1, 517 (2007).

[19] T. Ando, J. Phys. Soc. Jpn. 54, 2671 (1985).

[20] C. Jirauschek, J. Appl. Phys. 122, 133105 (2017).

[21] R. Terazzi, T. Gresch, M. Giovannini, N. Hoyler, N. Sekine, and J. Faist, Nat. Phys. 3, 329 (2007).

[22] N. Opačak and B. Schwarz, Phys. Rev. Lett. 123, 243902 (2019).

[23] Y. Bidaux, I. Sergachev, W. Wuester, R. Maulini, T. Gresch, A. Bismuto, S. Blaser, A. Muller, and J. Faist, Opt. Lett. 42, 1604 (2017).

[24] J. Hillbrand, P. Jouy, M. Beck, and J. Faist, Opt. Lett. 43, 1746 (2018).

[25] M. Piccardo, B. Schwarz, D. Kazakov, M. Beiser, N. Opačak, Y. Wang, S. Jha, J. Hillbrand, M. Tamagnone, W. T. Chen, A. Y. Zhu, L. L. Columbo, A. Belyanin, and F. Capasso, Nature (London) 582, 360 (2020).

[26] B. Meng, M. Singleton, M. Shahmohammadi, F. Kapsalidis, R. Wang, M. Beck, and J. Faist, Optica 7, 162 (2020).

[27] T. J. Kippenberg, R. Holzwarth, and S. A. Diddams, Science 332, 555 (2011).

[28] See Supplemental Material at http://link.aps.org/ supplemental/10.1103/PhysRevLett.127.093902 for the derivation and physical interpretation of the generalized optical susceptibility and laser master equation which include the Bloch gain, and additional numerical results, which includes Ref. [29].

[29] K. Shimoda, Introduction to Laser Physics (Springer, Berlin, Heidelberg, 1984).

[30] A. Wittmann, Y. Bonetti, J. Faist, E. Gini, and M. Giovannini, Appl. Phys. Lett. 93, 141103 (2008). 
[31] C. Henry, IEEE J. Quantum Electron. 18, 259 (1982).

[32] R. P. Green, J.-H. Xu, L. Mahler, A. Tredicucci, F. Beltram, G. Giuliani, H. E. Beere, and D. A. Ritchie, Appl. Phys. Lett. 92, 071106 (2008).

[33] L. Jumpertz, F. Michel, R. Pawlus, W. Elsässer, K. Schires, M. Carras, and F. Grillot, AIP Adv. 6, 015212 (2016).

[34] N. Opačak, F. Pilat, D. Kazakov, S. Dal Cin, G. Ramer, B. Lendl, F. Capasso, and B. Schwarz, Optica (2021), https:// doi.org/10.1364/OPTICA.428096.

[35] M. Piccardo, P. Chevalier, B. Schwarz, D. Kazakov, Y. Wang, A. Belyanin, and F. Capasso, Phys. Rev. Lett. 122, 253901 (2019).

[36] G. P. Agrawal, J. Opt. Soc. Am. B 5, 147 (1988).

[37] A. L. Gaeta, M. Lipson, and T. J. Kippenberg, Nat. Photonics 13, 158 (2019).

[38] T. W. Hänsch, Rev. Mod. Phys. 78, 1297 (2006).

[39] J. L. Hall, Rev. Mod. Phys. 78, 1279 (2006).

[40] F. Keilmann, C. Gohle, and R. Holzwarth, Opt. Lett. 29, 1542 (2004).
[41] A. Hugi, G. Villares, S. Blaser, H. C. Liu, and J. Faist, Nature (London) 492, 229 (2012).

[42] B. Schwarz, J. Hillbrand, M. Beiser, A. M. Andrews, G. Strasser, H. Detz, A. Schade, R. Weih, and S. Höfling, Optica 6, 890 (2019).

[43] J. Hillbrand, D. Auth, M. Piccardo, N. Opačak, E. Gornik, G. Strasser, F. Capasso, S. Breuer, and B. Schwarz, Phys. Rev. Lett. 124, 023901 (2020).

[44] L. A. Sterczewski, C. Frez, S. Forouhar, D. Burghoff, and M. Bagheri, APL Photonics 5, 076111 (2020).

[45] M. Singleton, P. Jouy, M. Beck, and J. Faist, Optica 5, 948 (2018).

[46] T. Herr, V. Brasch, J. D. Jost, C. Y. Wang, N. M. Kondratiev, M. L. Gorodetsky, and T. J. Kippenberg, Nat. Photonics 8, 145 (2014).

[47] I. S. Aranson and L. Kramer, Rev. Mod. Phys. 74, 99 (2002).

[48] L. Columbo, M. Piccardo, F. Prati, L. Lugiato, M. Brambilla, A. Gatti, C. Silvestri, M. Gioannini, N. Opačak, B. Schwarz, and F. Capasso, Phys. Rev. Lett. 126, 173903 (2021). 